\title{
EXPERIMENTOS INTELECTUALES CON MÁQUINAS: A PROPÓSITO DEL UTILITARISMO Y LA TEORÍA DEONTOLÓGICA EN EL ANÁLISIS DE LAS TEORÍAS DE LA JUSTICIA
}

Intellectual experiments with machines: on utilitarianism and deontological theory in the analysis of justice theories

\section{RESUMEN}

Este trabajo recoge una experiencia de innovación docente implementada en la asignatura de Filosofía del Derecho, partiendo de los contenidos impartidos sobre el utilitarismo y la teoría deontológica. Entre los objetivos que se pretenden están los de clarificar contenidos, proporcionar un aprendizaje duradero, y motivar la reflexión y el pensamiento crítico en el alumnado. Las acciones se desarrollan principalmente en el ámbito práctico, a partir de determinadas situaciones recogidas en escenas cinematográficas, en las cuales los avances tecnológicos suscitan varios dilemas. El alumnado debe realizar un diagnóstico de estas circunstancias, localizar en ellas la teoría o teorías de la justicia en juego, y adoptar una toma de postura. De este desarrollo se derivan varias críticas dirigidas a las teorías de la justicia en cuestión; se trata de objeciones que el alumnado debe constatar y que pasan a formar parte de los contenidos de la asignatura. Los resultados obtenidos son satisfactorios en términos generales; así lo confirma una encuesta realizada por el alumnado. Como conclusión cabría señalar que el nivel de consecución de los objetivos establecidos invita a seguir implementando las acciones en el futuro, siendo ello una oportunidad para mejorarlas con otros aspectos que pueden contribuir a completar la formación.

\section{PALABRAS CLAVE}

Filosofía del Derecho, utilitarismo, teoría deontológica, experimentos intelectuales, reflexión, pensamiento crítico. 


\section{ABSTRACT}

This paper describes a teaching innovation experience implemented in the subject Philosophy of Law, based on the content taught on utilitarianism and deontological theory. The objectives were, amongst others, to clarify the content, provide lasting learning, and prompt reflection and critical thinking in students. The actions were mainly carried out in the practical sphere, using situations depicted in film scenes in which technological advances pose various dilemmas. Students were asked to diagnose these circumstances, identify the theory or theories of justice at play in them, and take a position. This exercise gave rise to various criticisms of the theories of justice in question; the students had to verify these objections, which thus became part of the subject's content. The results obtained were generally satisfactory, as confirmed by a subsequent student survey. The level of achievement of the defined objectives suggests that the actions should continue to be implemented in future, offering the opportunity to improve them with other aspects that could help complete the training.

\section{KEYWORDS}

Philosophy of Law, utilitarianism, deontological theory, intellectual experiments, reflection, critical thinking. 
Sumario: 1. Introducción. 2. Contexto docente. 3. El utilitarismo y la teoría deontológica. 4. Objetivos. 5. El desarrollo de la experiencia. 5. 1. Aspectos preliminares. 5. 2. Ámbito práctico. 5. 2. a. Una máquina para proporcionar experiencias de felicidad. 5. 2. b. Una máquina para salvar muchas vidas en la II Guerra Mundial. 5. 2. c. Una máquina para evitar futuros atentados terroristas. 5. 3. Aportaciones del alumnado para el ámbito teórico: críticas a las teorías de la justicia. 6. Evaluación. 7. Resultados. 8. Conclusiones y prospectiva. 9. Bibliografía.

\section{Introducción.}

En el ámbito de la investigación iusfilosófica suele ser frecuente que después de la publicación de un estudio aparezca una réplica al mismo, y que probablemente esta última reciba también una respuesta con objeciones a los argumentos planteados. Este ejercicio ha dado lugar a debates que hoy son ineludibles en el estudio de la materia. En ocasiones, incluso las pretensiones críticas han aparecido ilustradas con la exposición de circunstancias que podrían tener lugar en la realidad, o bien que pertenecen a la ficción, pero que en todo caso se presentan con la intención de constatar que precisamente en aquellas recreaciones, se pone en cuestión un planteamiento determinado. A ello nos referimos con la expresión de "experimento intelectual".

A nuestro modo de ver, la extrapolación del ejercicio anterior al ámbito docente puede tener mucho sentido; de hecho, desde el punto de vista metodológico podría decirse que la propuesta encuentra puntos en común con el método del caso y el de resolución de problemas. Sustentamos la afirmación relativa a la conveniencia citada en los siguientes presupuestos: la misma podría servir para promocionar competencias tales como la capacidad reflexiva o el pensamiento crítico, y probablemente también para contribuir a una mejor comprensión de la asignatura y a la adquisición de un aprendizaje duradero en el tiempo.

Partiendo del planteamiento anterior, junto con otros contenidos, diseñamos un proyecto de innovación docente, concedido posteriormente por la Universidad de Alcalá ${ }^{1}$, que ha contextualizado la adaptación que venimos presentando en la asignatura de Filosofía del Derecho, a propósito del utilitarismo y la teoría deontológica. Las acciones desarrolladas en tal sentido se han centrando principalmente en el ámbito práctico, partiendo de diferentes situaciones recogidas en breves escenas cinematográficas que comparten una misma temática: las posibilidades que pueden ofrecer los avances tecnológicos. Más allá de lo sugestiva que pudiera considerarse la temática en lo que concierne a la consecución de las metas expuestas anteriormente, su tratamiento viene también motivado por las posibilidades que actualmente está abriendo la ciencia, de cara a un futuro más o menos inmediato. En principio, en ello debería verse un signo de progreso, pero el recorrido en tal dirección ineludiblemente debe ir acompañado de la reflexión acerca de lo que es justo; de otro modo el avance derivará en retroceso. Por lo demás, debemos adelantar que el trabajo realizado ha ido más allá de la parcela práctica, aprovechando las acciones desarrolladas en dicho contexto para incidir también en el ámbito de la teoría.

En las siguientes páginas nos referiremos con más detenimiento al desarrollo de la experiencia, pero antes de ello, y con la intención de contextualizarla, presentamos algunos

\footnotetext{
${ }^{1}$ El título y la referencia del proyecto de innovación docente son: Experimentos intelectuales con máquinas: a propósito del utilitarismo y la teoría deontológica (UAH/EV1166). Presenté una comunicación oral en el XIII Encuentro de Innovación en Docencia Universitaria de la Universidad de Alcalá (celebrado los días 1 y 2 de junio de 2021), en la cual resumí la experiencia docente que desarrollo en este trabajo.
} 
apartados previos en los que ampliamos los contenidos relativos al entorno docente en el que se han implementado las acciones, el marco temático de la asignatura a partir del cual han tenido lugar, y los objetivos que hemos pretendido con todo ello. De otro lado, una vez presentadas las acciones desarrolladas ofrecemos un espacio dedicado a su evaluación, y otro que recoge los resultados obtenidos, planteado a partir de una encuesta realizada por el alumnado. El trabajo termina con unas conclusiones finales y varias propuestas de carácter prospectivo, con las que pensamos que podría mejorarse la implementación para próximas ediciones.

Las acciones llevadas a cabo encuentran inspiración en aquellos ejercicios ideados con ánimo crítico, a los que nos referíamos al comienzo de esta introducción. Sin embargo, debemos destacar el carácter innovador de la labor realizada, pues con ella se adapta el planteamiento anterior al ámbito docente de la Filosofía del Derecho, recurriendo para ello a determinadas situaciones y actividades diseñadas a partir de aquellas que, al menos hasta donde llegamos a conocer, no se han considerado en la didáctica que nos ocupa.

\section{Contexto docente.}

La experiencia docente que presentamos se ha llevado a cabo en dos grupos de la asignatura de Filosofía del Derecho, que se imparte en el primer curso del Grado en Derecho, en la Facultad de Derecho de la Universidad de Alcalá. La didáctica se desarrolla a lo largo del segundo cuatrimestre del curso académico y, por tanto, las acciones se han implementado en este periodo cronológico. Su preparación tuvo lugar durante el primer cuatrimestre; fue entonces cuando diseñamos de forma pormenorizada las actividades que debería llevar a cabo el alumnado, partiendo del esquema cronológico bosquejado en un principio.

La asignatura puede cursarse conforme a dos modalidades, con evaluación continua o con evaluación final. En este último caso debe contarse con la autorización del Decanato de la Facultad de Derecho, y el alumnado tiene que realizar dos exámenes, uno teórico y otro práctico, sin tener que asistir a clase. En el caso de la evaluación continua el alumnado debe asistir a las clases, que se distribuyen en teóricas (impartidas los lunes) y prácticas (impartidas los miércoles). En esta modalidad la parte práctica se evalúa con la participación en las sesiones y con la realización de tres pruebas. De otro lado, la parte teórica se evalúa con un examen final. La experiencia que presentamos en este trabajo se ha desarrollado en el contexto de esta última modalidad de evaluación, conforme a la cual ha cursado la asignatura la mayoría del alumnado; concretamente, el $96 \%$ en un grupo, y el $97 \%$ en el otro.

En lo que respecta a la modalidad en la que se ha impartido la docencia, debe señalarse que dadas las circunstancias de pandemia en las que se ha desarrollado el curso, las clases han tenido lugar de forma semipresencial, siguiendo el Plan de actuación establecido por la Universidad ${ }^{2}$. Concretamente, la teoría se ha impartido online, por medio de clases desarrolladas con la plataforma Blackboard Collaborate del aula virtual de la asignatura. De otro lado, las clases prácticas han sido presenciales y, por tanto, en ellas hemos observado las medidas de seguridad establecidas a consecuencia de la situación citada anteriormente ${ }^{3}$.

\footnotetext{
${ }^{2}$ UNIVERSIDAD DE ALCALÁ (s. d.), Plan de actuación de la Universidad de Alcalá para la adecuación de la actividad universitaria en el curso 2020-2021, pp. 1, 2, [en línea] https://www.uah.es/export/sites/uah/.galleries/documentos/Plan-de-Actuacion_Curso-2020-2021.pdf. ${ }^{3}$ Se recogen gráficamente en el siguiente documento: UNIVERSIDAD DE ALCALÁ (s. d.), Medidas de prevención COVID-19 Coronavirus. Protégete y protege a los demás, [en línea] https://derecho.uah.es/facultad/documentos/medidas-prevencion-COVID.pdf.
} 
También atendiendo a las mismas circunstancias, las tutorías se han desarrollado online. A lo largo del curso y, por tanto, también en lo que respecta a las acciones que presentamos en este trabajo, hemos recurrido a aquellas para resolver posibles dudas.

\section{El utilitarismo y la teoría deontológica.}

En términos generales, podemos decir que los temas de los que se ocupa la Filosofía del Derecho son los que preocupan a la filosofía en general: el problema de la posibilidad y límites del conocimiento; el de la realidad, que remite a lo que hay en el mundo y sus orígenes; y el problema moral, que aborda la cuestión de cómo hemos de comportarnos ${ }^{4}$. La extrapolación de este esquema al ámbito iusfilosófico nos lleva a hablar de epistemología jurídica, que se refiere al problema de los tipos de conocimiento sobre el Derecho; ontología jurídica, que remite al estudio del 'ser' del Derecho; y axiología jurídica, que considera el "deber ser" de aquel ${ }^{5}$. En todo caso, junto con ello debe destacarse la importancia fundamental que tiene el último ámbito; una parcela que, a fin de cuentas, remite a una toma de postura en lo que respecta a la justicia ${ }^{6}$.

El temario de la asignatura en la que se han implementado las acciones contempla los tres problemas citados, pero del mismo modo, conviene también constatar que, en consonancia con el planteamiento expuesto, la cuestión de la justicia tiene un protagonismo indudable, apreciable tanto cuantitativa como cualitativamente. En este marco, entre otros contenidos la didáctica comprende diferentes teorías de la justicia, tanto clásicas como contemporáneas que, a su vez, remiten a las primeras; bien porque encuentran sus fundamentos en ellas, bien con ánimo de criticarlas.

La experiencia que presentamos se contextualiza en el ámbito temático recientemente bosquejado, concretamente en lo que respecta al utilitarismo y a la teoría deontológica. En el primer caso nos encontramos con una ética consecuencialista, que se desarrolla a partir del principio de utilidad, según el cual la acción justa es aquella que proporciona mayor felicidad para quienes viven en la sociedad ${ }^{7}$. En su versión tradicional benthamiana, el principio en cuestión se formula como "el mayor bien (equivalente a placer) para el mayor número" 8 . Contemporáneamente, siguiendo a Kymlicka, cabría añadir que se han venido considerando varias posiciones en torno a esta ética. Así, por ejemplo, el "hedonismo del bienestar", que considera como principal bien para la persona la experiencia que aporta placer; o la "utilidad no hedonista de los estados mentales", desde donde se afirma que hay otras experiencias con valor, que van más allá del placer, como, por ejemplo, la de escribir un poema ${ }^{9}$.

Por otra parte, en lo que respecta a la teoría deontológica exponemos el pensamiento kantiano, que se enfrenta a la apuesta consecuencialista del utilitarismo. En este caso se establece que debe obrarse por "buena voluntad", es decir, por deber, siguiendo lo que dicta la razón práctica. De este modo, si se quiere actuar de forma justa debe seguirse el imperativo categórico, en base al cual hay que proceder "según una máxima que pueda

\footnotetext{
4 GARCÍA SAN MIGUEL, L. (2003), Los fundamentos del Derecho (penúltimos apuntes). Madrid: Dykinson, p. 17.

${ }^{5}$ FARIÑAS, M․ J. (2000), "El conocimiento científico del derecho”, en Peces-Barba, G., Fernández, E., de Asís, R.: Curso de Teoría del Derecho. Madrid: Marcial Pons, segunda edición, pp. 137-139.

${ }^{6}$ GARCÍA SAN MIGUEL, L. (2003), p. 35.

${ }^{7}$ ZAPATERO, V., GARRIDO GÓMEZ, M. ${ }^{a}$ I., ARCOS RAMÍREZ, F. (2010), El Derecho como proceso normativo. Lecciones de Teoría del Derecho. Alcalá de Henares: Servicio de Publicaciones. Universidad de Alcalá, segunda edición, p. 224.

${ }^{8}$ GARCÍA SAN MIGUEL, L. (2003), p. 192.

${ }^{9}$ ZAPATERO, V., GARRIDO GÓMEZ, M. ${ }^{a}$ I., ARCOS RAMÍREZ, F. (2010), p. 226.
} 
valer a la vez como ley universal". Ello, siguiendo la segunda formulación de dicho imperativo, obliga a tratar a las personas como un fin en sí mismas y no como un medio. En atención a lo dicho, entre las implicaciones de este planteamiento encontramos la prohibición de aquellas acciones que no puedan tomarse como principios morales universalizables ${ }^{10}$; así acontece, por ejemplo, con la mentira, pues de lo contrario sería imposible el trato entre personas ${ }^{11}$.

\section{Objetivos.}

Con la experiencia que venimos considerando hemos pretendido alcanzar los objetivos que aparecen en la siguiente relación.

1. Presentar al utilitarismo y a la teoría deontológica como teorías de la justicia que tienen una proyección práctica. Con este objetivo pretendemos ofrecer una percepción pragmática de los contenidos impartidos, alejada de aquella concepción que ha venido anclando a la iusfilosofía en lo abstracto.

2. Motivar al alumnado con las teorías de la justicia en cuestión, valiéndonos para ello de recursos sugestivos. No pretendemos únicamente constatar la dimensión práctica de la Filosofía del Derecho, sino también que el proceso que conduzca a su confirmación resulte sugerente para el alumnado, y contribuya a la consecución de otros objetivos que consideramos en lo que sigue.

3. Renovar un ejercicio que resulta ineludible para quienes van a ser futuros/as juristas, y del cual la Filosofía del Derecho debe encargarse de manera especial: reflexionar.

4. Facilitar la comprensión de los contenidos impartidos sobre el utilitarismo y la teoría deontológica. Su mera exposición teórica podría suscitar numerosas dudas y problemas de comprensión entre el alumnado, y la puesta en práctica puede resultar un recurso idóneo para atajar dichas circunstancias.

5. Facilitar el aprendizaje duradero de los contenidos relativos al utilitarismo y la teoría deontológica. No se pretende transmitir conocimientos cuya retención únicamente alcance a las fechas de las pruebas evaluables o al examen final; la intención es aportar una experiencia capaz de proporcionar un aprendizaje prolongado en el tiempo.

6. Entrenar la capacidad de análisis reparando en los detalles. En muchas ocasiones, cuando trabajamos en el ámbito jurídico los pormenores marcan la diferencia, y el planteamiento de la actividad docente en este sentido puede contribuir a formar profesionales más competentes.

7. Fomentar la capacidad crítica con respecto a las teorías de la justicia que se pretenden abordar. Este objetivo enlaza con la vertiente axiológica de la Filosofía del Derecho, que insta a formar a estudiantes cuyas competencias trasciendan del mero conocimiento de las normas, para centrar la atención en el tema de la justicia. En ello va también la intención de proporcionar recursos que contribuyan a enriquecer el punto de vista particular de cada persona sobre la justicia.

\footnotetext{
${ }^{10}$ ZAPATERO, V., GARRIDO GÓMEZ, M. ${ }^{\mathrm{a}}$ I., ARCOS RAMÍREZ, F. (2010), pp. 232-235.

${ }^{11}$ GARCÍA SAN MIGUEL, L. (2003), p. 153.
} 
8. Tratar de ofrecer una aproximación al nivel de consecución de varios de los objetivos considerados en los puntos precedentes.

\section{El desarrollo de la experiencia 5. 1. Aspectos preliminares}

Las acciones llevadas a cabo parten de los contenidos impartidos sobre el utilitarismo y la teoría deontológica, y como ya adelantamos, se han implementado principalmente en el ámbito práctico, aunque no exclusivamente. Las prácticas se desarrollaron de forma presencial, siguiendo el sistema de desdoble de grupos establecido por la Facultad de Derecho, según el cual cada grupo se divide en dos subgrupos. De este modo, las prácticas se llevaron a cabo en cuatro subgrupos, dado que, como ya señalamos, la experiencia en cuestión se ha implementado en dos grupos. Este sistema encuentra su motivación en razones pedagógicas, pues una ratio menor de alumnado da la posibilidad de ofrecer un seguimiento más cercano y detenido; un aspecto que resulta especialmente necesario en el ámbito práctico. Además de ello, los desdobles también han facilitado el desarrollo de las clases atendiendo a las medidas de distancia entre personas, que hemos debido observar a consecuencia del COVID-19.

Desde el punto de vista metodológico, el planteamiento seguido guarda conexión con el método del caso ${ }^{12}$, una estrategia que consiste en presentar una situación que pudiera darse en la realidad, y que solicita una toma de postura en la que han de tenerse en cuenta las consecuencias de la decisión que se tome ${ }^{13}$. No obstante, cabría apuntar que las acciones propuestas han ido más allá de dicho esquema y que, al menos a día de hoy, algunas de las circunstancias analizadas pertenecen al ámbito de la ficción. En el sentido expuesto, la experiencia se distancia además del método del caso entendido como análisis jurisprudencial ${ }^{14}$. Por lo demás, el componente reflexivo que incluyen las actividades planteadas permite afirmar que las mismas guardan relación con el método de resolución de problemas ${ }^{15}$.

Concretamente, las acciones se implementaron en dos prácticas. Seis días antes de que se realizase cada una de ellas, el alumnado tuvo disponible en el aula virtual de la asignatura el enunciado de la práctica que se llevaría a cabo en la semana siguiente. El documento debía descargarse con vistas a revisar de forma individual las cuestiones planteadas. Ya en la clase práctica, comenzamos analizando los dilemas que se plantean en situaciones recogidas en breves escenas cinematográficas, previa exposición de los datos principales de la obra que las contextualizaba, así como del argumento en el que se

\footnotetext{
12 Sobre la implementación del mismo en el ámbito de la Filosofía del Derecho puede consultarse GARRIDO GÓMEZ, M. ${ }^{a}$ I. (2013), "La innovación en la docencia y el aprendizaje de la Filosofía del Derecho por medio del método de casos", REJIE: Revista Jurídica de Investigación e Innovación Educativa, $\quad 8, \quad$ pp. $\quad 25-42, \quad$ [en $\quad$ línea] https://revistas.uma.es/index.php/rejienuevaepoca/article/view/7749/7263.

${ }^{13}$ MARGALEF GARCÍA, L. (s. d.), Estrategias metodológicas. [Alcalá de Henares]: Vicerrectorado de Planificación Académica y Profesorado. Dirección de Formación del Profesorado Universitario. Universidad de Alcalá. [Dosier], pp. 25-28; CABELLOS CASTILLA, M. ${ }^{a}$ R., CASTILLO GARCÍA, G. S., TORNÉ VALLE, E. (2007), “Para qué y cómo innovar en el aula universitaria?”, en Margalef García, L., Torné Valle, E. (eds.): Estrategias de innovación docente para favorecer el aprendizaje autónomo de los estudiantes de la Universidad de Alcalá. Alcalá de Henares: Servicio de Publicaciones Universidad de Alcalá, p. 52.

${ }^{14}$ Sobre la metodología entendida en tal sentido RUIZ RESA, J. D. (2017), Innovación docente y nuevas tecnologías. El derecho en la economía del aprendizaje. Valencia: Tirant lo Blanch, pp. 220-222.

${ }^{15}$ Sobre dicha metodología RUIZ RESA, J. D. (2017), p. 77.
} 
desarrollaba la trama en líneas generales. A partir de los casos presentados llevamos a cabo puestas en común sobre las cuestiones solicitadas, con vistas a que el alumnado interviniese con sus aportaciones. A ello nos vamos a referir de forma más pormenorizada en los siguientes apartados. Del mismo modo, reservamos el último espacio del desarrollo que presentamos para las acciones contextualizadas en el plano teórico.

\section{2. Ámbito práctico}

\section{2. a. Una máquina para proporcionar experiencias de felicidad}

La primera práctica se desarrolló una vez impartida la teoría sobre el utilitarismo. En ella analizamos determinadas situaciones que presentan escenas de dos películas, a las que nos vamos a referir en este espacio y en el siguiente.

Por una parte, consideramos algunos extractos de Matrix ${ }^{16}$. Su argumento descubre un mundo devastado por las máquinas, que vencieron la guerra contra las personas. Desde entonces, estas últimas están sometidas a aquellas, y viven en un estado vegetativo. Sin embargo, las máquinas han creado una realidad virtual llamada Matrix; un programa informático con el cual hacen posible que las personas sientan que viven en un mundo normal, que pueden disfrutar de una buena comida, ser ricas, famosas, etc. Con ello Matrix hace posible el principio de utilidad; la mayor felicidad para el máximo número de personas. Se trata de un argumento que ineludiblemente remite al experimento intelectual de "La máquina de experiencias" de Robert Nozick ${ }^{17}$, recogido en su libro Anarquía, Estado y Utopía con el fin de criticar al utilitarismo ${ }^{18}$.

El alumnado debe establecer una relación entre el argumento considerado de Matrix y "La máquina de experiencias" de Robert Nozick, de cuyo texto se aporta un extracto para su análisis en el enunciado de la práctica. Además, también tiene que identificar cómo se manifiesta el utilitarismo en dichos casos, y determinar si se está o no de acuerdo con las circunstancias analizadas, señalando con ello si la postura expuesta se desarrolla o no en la línea del utilitarismo. En todo caso, debe justificarse la respuesta.

Si se aboga por las experiencias de felicidad, posibles gracias a Matrix o a la máquina ideada por Robert Nozick, se está argumentando en el marco del utilitarismo. Por otra parte, la ética consecuencialista quedaría cuestionada -y más en concreto las versiones de la misma que expusimos anteriormente- si se argumenta que en la vida hay otras cosas, más importantes que el placer o determinados estados mentales, como, por ejemplo, tener una vida real, que sea verdad, en definitiva ${ }^{19}$. En este caso se apostaría por el simple hecho de vivir la vida, aun cuando ello suponga afrontar el dolor y la tristeza que aquella conlleva. Se trata de la línea argumental que puede verse en la intención crítica que ofrece Robert Nozick con su experimento intelectual. Sin duda, el planteamiento tiene un peso significativo, pero no parece exento de réplica; no al menos si se piensa que determinadas circunstancias que presenta la vida pueden suponer obstáculos difícilmente superables, frente a los cuales cabría considerar la opción de bienestar que ofrecen las máquinas, como una posible vía de evasión ante dichas situaciones.

\footnotetext{
${ }^{16}$ WACHOWSKI, J. S., WACHOWSKI, L. (dir.) (1999), Matrix. Warner Bros.

${ }^{17}$ WACHOWSKI, J. S., WACHOWSKI, L. (dir.) (2004), The Roots of the Matrix. Warner Bros.

${ }^{18}$ NOZICK, R. (1974), Anarquía, Estado y utopía. México: Fondo de Cultura Económica, pp. 53-56. Sobre la cuestión ZAPATERO, V., GARRIDO GÓMEZ, M. ${ }^{a}$ I., ARCOS RAMÍREZ, F. (2010), p. 226.

19 A estos argumentos se refiere Christopher Grau, Assistant Professor of Philosophy de la Florida International University, en WACHOWSKI, J. S., WACHOWSKI, L. (dir.) (2004).
} 


\section{2. b. Una máquina para salvar muchas vidas en la II Guerra Mundial}

En segundo lugar, nos detenemos en un dilema que aparece en la película The Imitation Game (Descifrando Enigma) ${ }^{20}$. En esta obra cinematográfica se narra la vida de Alan Turing, un lógico matemático que inventó una máquina para descifrar los mensajes del ejército nazi durante la II Guerra Mundial. Tal sistema, por tanto, daba lugar a evitar o repeler los ataques del enemigo, salvándose con ello muchas vidas. No obstante, el descubrimiento tenía un inconveniente: el hecho de abortar los ataques de forma indiscriminada delataría el invento de Alan Turing, y tras tener conocimiento del mismo el ejército nazi buscaría otros cauces para transmitir la información, impidiendo así su detección. El resultado de ello sería que no se podrían evitar otras muchas muertes en lo sucesivo.

A partir de la contextualización anterior, el dilema que nos interesa tratar es el siguiente. La máquina de descifrado intercepta información sobre un ataque de submarinos a un convoy de barcos ingleses. En uno de esos barcos viaja el hermano de una de las personas que forma parte del equipo de investigación de Alan Turing. La postura de este último es no actuar, basándose en la argumentación expuesta al final del párrafo precedente. Por otra parte, el miembro del equipo de Alan Turing se enfrenta a la decisión planteada por este, pues quiere salvar a su hermano que viaja en uno de los barcos del convoy que va a ser atacado ${ }^{21}$.

En esta ocasión el alumnado debe determinar quién es utilitarista en la situación expuesta, si Alan Turing o la persona que trabaja en su equipo de investigación. La postura del primero es la que se enmarca en la teoría de la justicia en cuestión, pues con su decisión pretende salvar muchas más vidas en el futuro. De otro lado, la acción de su compañero de trabajo se enfrenta a dicha ética, dado que su intención es salvar a un ser querido, con independencia del resultado que pretende evitar Alan Turing.

Por otra parte, debe responderse a la pregunta de qué se hubiera hecho en el caso sometido a análisis, si la decisión adoptada se enmarca o no en el utilitarismo, y las razones que sustentan dicho proceder. $\mathrm{Si}$, como ya señalamos, se decide no abortar el ataque para salvar muchas más vidas en el futuro, se estaría actuando en la línea del utilitarismo. De otro lado, este último quedaría cuestionado si se responde abogando por la salvación del ser querido, aun a consecuencia de que ello imposibilite el resultado anterior.

\section{2. c. Una máquina para evitar futuros atentados terroristas}

En la semana siguiente a aquella en la que realizamos la práctica que recogió las acciones precedentes, llevamos a cabo otra, una vez explicada la teoría deontológica. En esta ocasión partimos de las circunstancias que aparecen en algunas escenas de la película Código fuente ${ }^{22}$. Su argumento presenta a un capitán gravemente herido, prácticamente muerto y sin posibilidad alguna de recuperación, que, no obstante, conserva cierta función de parte de su cerebro. Esta circunstancia se aprovecha para poner en práctica un proyecto secreto basado en un programa informático, mediante el cual una capitana se comunica con el militar anterior y este es enviado a una realidad paralela para descubrir a un

\footnotetext{
${ }^{20}$ TYLDUM, M. (dir.) (2015), The Imitation Game (Descifrando Enigma). Black Bear Pictures.

${ }^{21}$ En la siguiente referencia, donde se constata el drama de la escena, se informa de que se trata de una situación inventada: SÁNCHEZ MUÑOZ, J. M. (2018), "Informe sobre la película «Descifrando Enigma»", Revista "Pensamiento Matemático", VIII / 2, p. 199, [en línea] https://dialnet.unirioja.es/servlet/articulo?codigo $=6636704$.

22 JONES, D. (dir.) (2011), Código fuente. Vendôme International.
} 
terrorista que cometerá un atentado de forma inminente. El terrorista es descubierto, se evita el atentado y, por tanto, se salvan muchas vidas. El superior que dirige el proyecto,

alentado por el éxito de la experiencia, acuerda programar otras intervenciones para el futuro, con el fin de conseguir resultados como el logrado. Por ello, ordena a la capitana que mantenga conectado al capitán, aun cuando este último, consciente de su situación, manifiesta que quiere la desconexión. La capitana responde al superior con un "sí señor", pero finalmente desconecta al militar.

A partir del planteamiento anterior el alumnado debe responder a la pregunta de con qué teoría de la justicia puede relacionarse la acción del superior que dirige el proyecto, y realizar el mismo ejercicio con respecto a la acción de la capitana que lleva a cabo la desconexión. De un lado, la postura del primero se desarrolla en la línea del utilitarismo, pues pretende evitar muchas muertes futuras, lo cual conlleva mayor felicidad para el mayor número de personas. De otro lado, la acción de la capitana no puede considerarse utilitarista, pues al desconectar al militar interrumpe la continuidad del proyecto y, por tanto, impide la obtención del resultado anterior.

En un primer acercamiento, el desarrollo expuesto quizá pudiera invitar a pensar que la capitana actúa en la línea de la teoría deontológica, si se interpreta que lo que pretende es evitar que se utilice a una persona como un medio para algo, y no como un fín en sí misma. Sin embargo, en la progresión de los hechos se aprecia que la capitana miente al superior del proyecto; recordemos que le confirma que va a mantener conectado al capitán, pero finalmente lo desconecta. Esta circunstancia queda fuera de la moral kantiana, pues desde esta perspectiva la mentira no puede considerarse un principio universalizable.

Después de responder a las cuestiones anteriores, y teniendo en cuenta todo el desarrollo expuesto, el alumnado debe abordar la pregunta de qué hubiera hecho en el caso analizado, y si su acción se desarrolla en la línea del utilitarismo, de la teoría kantiana, o si no se identifica con ninguna de las dos.

\section{3. Aportaciones del alumnado para el ámbito teórico: críticas a las teorías de} la justicia

Una vez realizadas las prácticas anteriores, y en base a las respuestas dadas a las cuestiones planteadas, en la clase teórica siguiente el alumnado participó exponiendo los aspectos críticos que podrían considerarse a propósito de cada una de las teorías de la justicia consideradas. Siguiendo el sistema de docencia establecido, al que nos referimos anteriormente en la contextualización de aquella, esta sesión se desarrolló de forma virtual. Para organizar las participaciones solicitamos que quien quisiera intervenir accionase la opción de pedir la palabra, disponible en la plataforma Blackboard Collaborate. Con este sistema el alumnado fue realizando aportaciones paulatinamente, bien por medio del micrófono, siempre que esta opción fue posible, o bien escribiendo en el chat disponible también en la aplicación de la sesión, en el caso de que no se tuviera la posibilidad de utilizar micrófono.

Concretamente, los contenidos que se establecieron fueron los siguientes. Por una parte, frente al utilitarismo puede decirse que experimentos como el que recrean Matrix y "La máquina de experiencias" invitan a pensar que es mejor vivir la vida real, tal cual es, con sus penas y tristezas, en vez de tener sensaciones de felicidad irreales y vacías. También a propósito de la misma teoría de la justicia, se planteó la objeción de que aquella conlleva la asunción de una imparcialidad que impide tener en cuenta las relaciones 
especiales que podamos tener con determinadas personas (familiares, amistades, etc.) ${ }^{23}$. Por último, quedó constatado que la ética consecuencialista que apuesta por el bienestar del mayor número puede dar lugar a la vulneración de los derechos de una persona.

De otro lado, en lo que respecta a la teoría deontológica kantiana se incidió en su carácter rígido, especificado en la exclusión de la mentira en cualquier situación. En este sentido, resulta representativo que en la segunda práctica realizada varias personas considerasen moral la forma de proceder de la capitana, algo que, además de constatar la objeción anterior, también parece evidenciar el carácter formalista y abstracto de la ética en cuestión, que no establece principios concretos que indiquen la forma en la que ha de actuarse ${ }^{24}$.

Con las participaciones anteriores fue el propio alumnado el que amplió los contenidos teóricos que debían tenerse en cuenta en la asignatura, a propósito de las teorías de la justicia con las que trabajamos, y que formaron también parte de la materia que había que preparar de cara al examen final de la asignatura, de carácter teórico.

\section{Evaluación}

Como ya adelantamos, la experiencia llevada a cabo se desarrolló en el marco de la evaluación continua, lo cual supone realizar tres pruebas a lo largo del cuatrimestre. Se trata de ejercicios prácticos que debían responderse de forma individual, por escrito y presencialmente, y que se basaron en las prácticas con las que trabajamos en las semanas previas a aquellas en las que tuvieron lugar las pruebas. Concretamente, en cada una de las pruebas se evaluaron 3 o 4 prácticas. Las cuestiones que incluyen estas pruebas pueden haberse realizado en las prácticas precedentes, tener alguna variación con respecto a los ejercicios desarrollados en aquellas, o bien ser distintas; si bien, en este último caso su resolución puede llevarse a cabo sin complicación, si se han comprendido los resultados a los que llegamos con las puestas en común planteadas en las clases prácticas. La nota obtenida por cada una de estas pruebas supone el $20 \%$ de la final, es decir, 2 puntos de la asignatura.

Las prácticas que comprendieron la experiencia que nos ocupa fueron objeto de evaluación en la segunda prueba realizada. La misma se evaluó sobre 10 puntos e incluyó tres preguntas referidas a las acciones que venimos considerando, en las cuales recogimos las situaciones analizadas, seguidas de una o varias cuestiones sobre aquellas. Ejemplos de estas últimas son los siguientes:

Sobre la primera situación.

- ¿Estás de acuerdo con la crítica que plantea la persona que escribió este texto ${ }^{25}$ ? Responde diciendo simplemente sí o no.

- Teniendo en cuenta la respuesta dada a la pregunta anterior, ¿se enmarca tu postura en el utilitarismo?, ¿por qué? Debes relacionar la respuesta a esta pregunta con el contenido del texto.

Sobre la segunda situación.

\footnotetext{
${ }^{23}$ Sobre estas críticas ZAPATERO, V., GARRIDO GÓMEZ, M. ${ }^{a}$ I, ARCOS RAMÍREZ, F. (2010), pp. $226,228$.

${ }^{24}$ Sobre ello ZAPATERO, V., GARRIDO GÓMEZ, M. ${ }^{a}$ I, ARCOS RAMÍREZ, F. (2010), pp. 236, 237; GARCÍA SAN MIGUEL, L. (2003), p. 153.

${ }^{25}$ Se trata de "La máquina de experiencias" de Robert Nozick.
} 
- ¿En qué teoría de la justicia puede enmarcarse la posición de Alan Turing? Justifica la respuesta.

-Plantea una crítica a la postura de Alan Turing, teniendo en cuenta el dilema suscitado en el texto.

Sobre la tercera situación.

- ¿Consideras que la acción de la capitana es utilitarista? Si la respuesta es afirmativa justifícala, comparando la acción de la capitana con la teoría deontológica. Si la respuesta es negativa explica cómo tendría que haber actuado la capitana para que su acción pudiera considerarse utilitarista?

Cada una de estas preguntas tuvo un valor de 1,5 puntos y, por lo tanto, en total supusieron 4,5 puntos sobre 10 . Si, como ya señalamos, tenemos en cuenta que la prueba supone 2 puntos de la asignatura, en el caso de haberse respondido correctamente a las preguntas incluidas en ella sobre la experiencia llevada a cabo, se habría obtenido casi un punto (9 décimas) de la nota final de la asignatura.

Del mismo modo, el alumnado tuvo también la oportunidad de participar en las clases prácticas a la hora de realizar las puestas en común, en relación a las solicitudes planteadas en cada caso, y también en lo que respecta a la clase teórica en la que establecimos las críticas dirigidas a cada una de las teorías de la justicia en cuestión. En estos casos las participaciones correctas supusieron un aumento de una o varias décimas en la nota obtenida en la prueba que consideramos anteriormente.

\section{Resultados}

Al final de curso invitamos al alumnado a que respondiese a una encuesta, en la que preguntamos sobre varios aspectos relacionados con las acciones realizadas. Llevamos a cabo su diseño recurriendo a la herramienta disponible para tal fin en la plataforma virtual de la asignatura, y el alumnado tuvo un plazo de dos semanas para responder a las cuestiones accediendo a la aplicación. En total, teniendo en cuenta los dos grupos en los que se desarrolló la experiencia, respondieron a la encuesta 41 personas, es decir, casi el $72 \%$ de alumnado que ha cursado la asignatura conforme a la evaluación continua.

Las preguntas que planteamos en la encuesta son las que presentamos a continuación, al pie de cada uno de los gráficos que muestran los resultados obtenidos en cada caso:

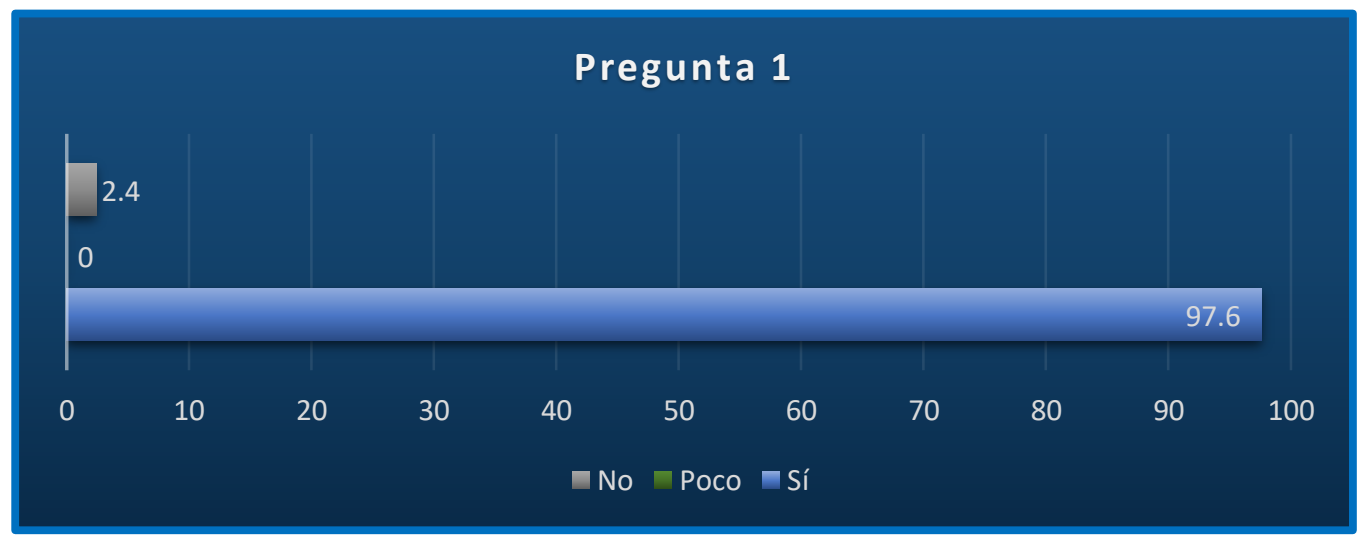


Gráfico 1. ¿Te han ayudado las prácticas realizadas a comprender mejor la teoría impartida?

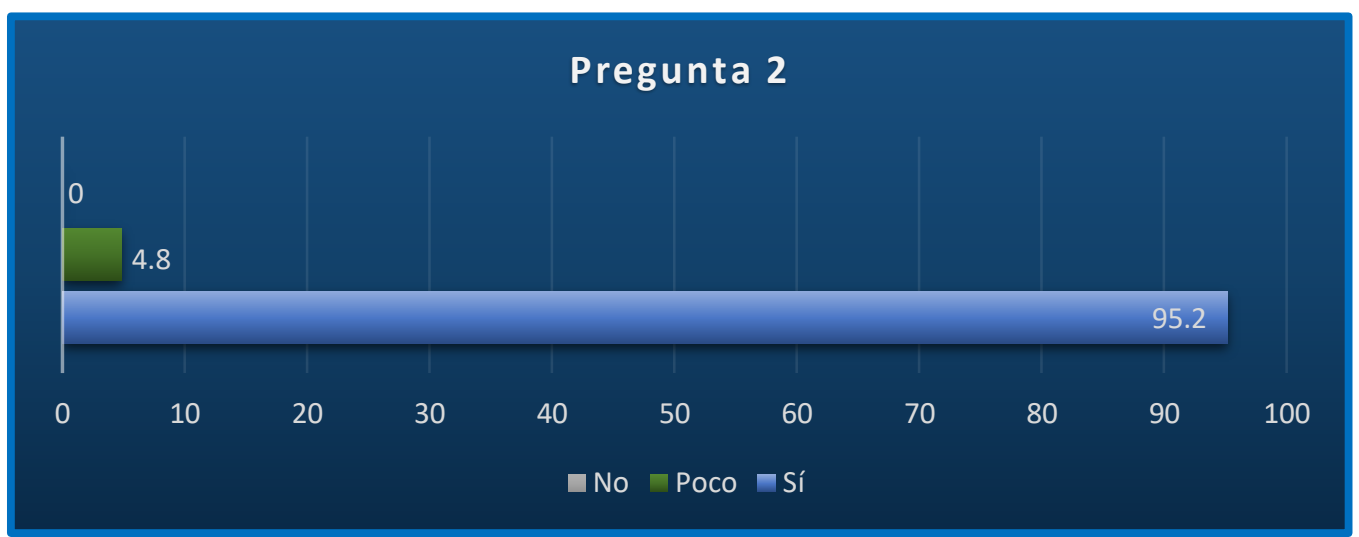

Gráfico 2. ¿Te han ayudado las prácticas realizadas a recordar la teoría impartida?

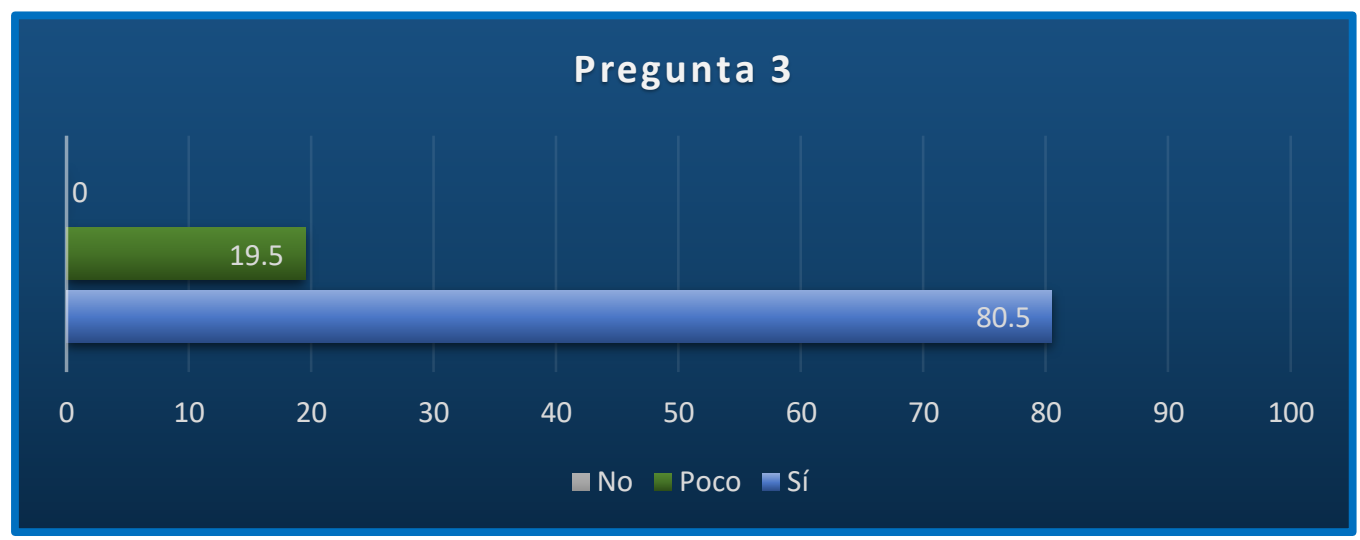

Gráfico 3. ¿Te han servido las prácticas para reflexionar?

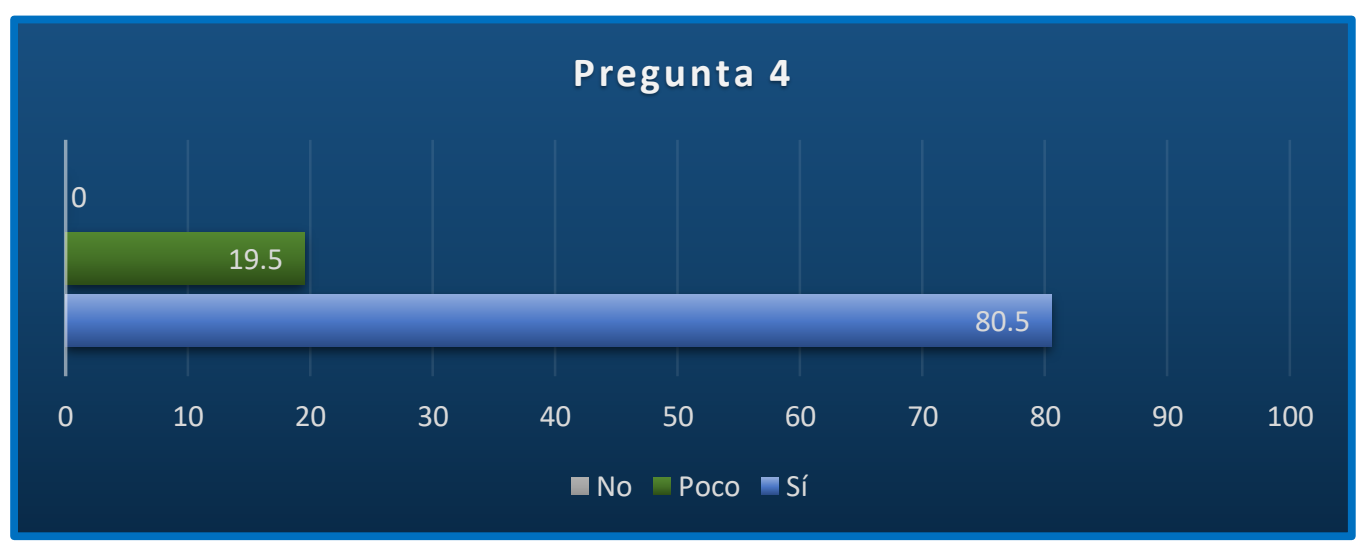

Gráfico 4. ¿Han contribuido las prácticas a enriquecer tu concepto de justicia? 


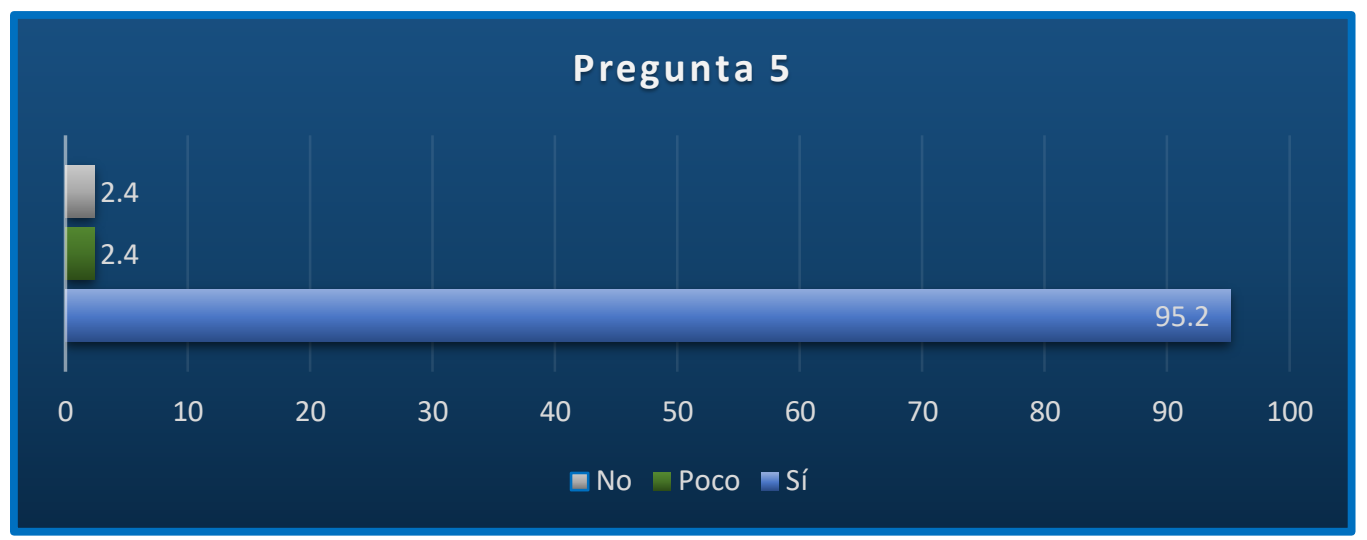

Gráfico 5 ¿Te ha resultado interesante la metodología de análisis de casos?

De los resultados que arrojan los gráficos anteriores resulta significativa la alta tasa de respuestas afirmativas que se observa en las preguntas 1, 2 y 5 . Merecen especial consideración las respuestas dadas a la primera de ellas. En este caso el 97,6\% del alumnado ha considerado que las acciones llevadas a cabo han hecho posible una mejor comprensión de la teoría impartida. A nuestro modo de ver, ello puede considerarse un logro que contribuye a diluir el aura de abstracción que en ocasiones se ha venido atribuyendo a la didáctica que contextualiza las acciones desarrolladas.

Del mismo modo, debe destacarse el resultado obtenido en la segunda pregunta; en ella un $95,2 \%$ del alumnado consideró que las prácticas ayudan a recordar la teoría impartida. Ello constata los efectos positivos de la experiencia realizada de cara a la preparación de la prueba evaluable y del examen final teórico. Además, el resultado invita a pensar que las acciones llevadas a cabo probablemente promocionarán el aprendizaje significativo, contribuyendo a que se recuerden los contenidos impartidos más allá del espacio cronológico que ha comprendido el desarrollo de la asignatura.

En lo que respecta a los resultados obtenidos en la pregunta 5, nos encontramos nuevamente con que, en un porcentaje muy significativo (un 95,2\%), el alumnado ha considerado interesante la metodología utilizada; algo que, entendemos, confirma que aquella sirve de reclamo para suscitar su atención y, por tanto, su implicación en las temáticas tratadas.

Consideramos también que debe destacarse el índice de respuestas afirmativas que se aprecia en las preguntas 3 y 4 , y que alcanza en ambos casos al 80,5\%. El resultado es relevante, pues informa de que más de tres cuartas partes del alumnado tiene una valoración positiva con respecto a las cuestiones planteadas. No obstante, también es cierto que en estos casos algunas personas marcaron la opción de 'poco'; concretamente, el $19,5 \%$ de quienes respondieron. Este dato, así como el relativo a la tasa de respuestas negativas perceptible en las preguntas 1 y 5 , ofrecen razones para la reflexión. A ello nos referiremos en el siguiente apartado.

\section{Conclusiones y prospectiva}

A la hora de plantear las acciones que hemos presentado en este trabajo partimos de una serie de presupuestos, de los cuales dejamos constancia en la introducción. Desde el comienzo supusimos que la adaptación del modelo del experimento intelectual al 
ámbito docente redundaría en algún sentido en aspectos tales como un mejor entendimiento de la teoría impartida, la transmisión de un aprendizaje significativo y la promoción de competencias relativas a la reflexión y al pensamiento crítico. De hecho, planteamos estos aspectos como objetivos a los que aspirábamos. No obstante, en la fase inicial de la experiencia desconocíamos el alcance que pudieran tener los resultados estimados; y por ello uno de los objetivos establecidos (el que aparece en último lugar en el apartado dedicado a aquellos) fue precisamente el de tratar de baremar su repercusión.

De entrada, debemos señalar que ya en el momento de la implementación de las acciones programadas, percibimos que las mismas reclamaban el interés de una parte considerable del alumnado. Más concretamente, si tenemos en cuenta los resultados que arroja la encuesta elaborada sobre la labor llevada a cabo, podemos afirmar que aquella supone una contribución significativa en lo que respecta a los aspectos señalados en el párrafo anterior, si bien la tasa de respuestas positivas desciende en dos casos.

No sabríamos determinar con certeza las razones de la circunstancia expuesta, pero consideramos que algunas de ellas podrían explorarse en los sentidos que se apuntan a continuación. Las preguntas en cuestión se refieren a las aportaciones que hayan podido proporcionar las prácticas de cara a la reflexión y al enriquecimiento del concepto de justicia personal. Quizá pueda haberse dado la circunstancia de que para las personas que respondieron 'poco' en estos casos, los dilemas planteados probablemente no se considerasen como tales, dado que, de entrada, se podría haber tenido una respuesta clara en relación a las situaciones tratadas. En este sentido, la consideración de opciones diferentes apenas habría dado lugar a la reflexión, habida cuenta de que no se tendría duda alguna en lo que respecta a la postura a adoptar en los casos analizados. De otro lado, cabría señalar que, al menos en cierta parte, los resultados que nos ocupan pudieran atribuirse a la desmotivación en lo que respecta al seguimiento de la evaluación continua; algo que quizá habría suscitado, no únicamente las respuestas que venimos considerando, sino también la elección de las opciones 'no' o 'poco' en las preguntas 1, 2 y 5 . Estas últimas no son especialmente representativas en los gráficos, pero están presentes.

En todo caso, consideramos que, en términos generales, las conclusiones expuestas invitan a seguir implementando las acciones consideradas en futuras ediciones de la asignatura. Ello, además, ofrece la oportunidad aportar posibles mejoras. En este sentido, pensamos que podría resultar interesante desarrollar acciones complementarias en torno a la teoría deontológica, que ha tenido una menor representación en la labor realizada. Por ello, y también con la intención de seguir aportando más argumentos para promocionar la formación y reflexión entre el alumnado, proponemos dos posibles cauces de desarrollo que apuntamos a continuación.

De un lado, puede resultar interesante reconsiderar el último caso con el que hemos trabajado, pues en él se encuentra latente el tema de la dignidad de la persona. Quizá, en un principio, podría plantearse la cuestión de si el hecho de desconectar al capitán del programa informático atenta contra su dignidad, dado que el resultado de ello es su muerte. Sin embargo, esta primera aproximación debe criticarse, pues el concepto de dignidad kantiano no conduce necesariamente a mantener con vida a la persona. Como se ha señalado por quienes estudian el pensamiento del autor, para él hay deberes situados por encima de la supervivencia, relacionados con la "propia estima"; deberes que además encuentran su fundamento en la dignidad. En este sentido, cabría afirmar que la cuestión 
no es alargar la vida, sino vivir con dignidad ${ }^{26}$. Iracheta Fernández, de quien tomamos estas ideas, escribe lo siguiente comentando el pensamiento kantiano, en lo que respecta a la eutanasia voluntaria ${ }^{27}$.

"Gracias al avance de la tecnología biomédica, a menudo las personas permanecen con vida por la simple finalidad de complacer intereses ajenos. En tales circunstancias el recurso de la eutanasia aparece como un deber moral por la razón de que los intereses de las personas involucradas se satisfacen a costa de la voluntad del paciente".

El argumento cobra especial relevancia en el caso que consideramos, pues la persona conectada al programa informático se encuentra en una situación irreversible y está siendo utilizada en contra de su voluntad.

De otro lado, con vistas a continuar con el objetivo anterior entendemos que podría resultar interesante incluir la cuestión de la verdad engañosa en el pensamiento kantiano, a la cual se refiere $\operatorname{Sandel}^{28}$. Desde esta perspectiva, aquella se diferencia de la mentira en que podría ser permisible desde el punto de vista moral. Su consideración supondría una aportación más en lo que respecta a los contenidos a tener en cuenta, pero también un recurso para analizar circunstancias que pueden ser perfectamente reales, como así lo constata el autor citado anteriormente ${ }^{29}$.

La adaptación de la propuesta a la acción desarrollada podría tener lugar mediante la formulación de la siguiente cuestión para el alumnado: ¿qué podría haber respondido la capitana al superior, para evitar el problema de la mentira en el planteamiento kantiano? Como posibles respuestas en este caso cabría señalar que en vez de responder "sí señor", podría, por ejemplo, haber dicho "voy a comprobar las circunstancias en la que se encuentra el capitán", o "tenemos que seguir con el proyecto". En el primer caso la respuesta no incide directamente en la orden dada por el superior, y en el segundo muestra la conformidad con seguir adelante con el proyecto, pero no con mantener al militar conectado; con lo cual podría llevarse a cabo la desconexión sin incurrir con ello en la mentira. Teniendo en cuenta la exposición de Sandel sobre el tema ${ }^{30}$, pensamos que en el ámbito docente este ejercicio puede considerarse una invitación para prestar atención a lo que se dice, reparando en todos los detalles; a fin de cuentas, una labor propia de quien ejerce la abogacía.

\section{Bibliografía.}

- CABEllos CASTIlla, M. ${ }^{a}$ R., CASTillo GARCÍA, G. S., TORNÉ VALLE, E. (2007), “Para qué y cómo innovar en el aula universitaria?”, en Margalef García, L., Torné Valle, E. (eds.): Estrategias de innovación docente para favorecer el aprendizaje autónomo de los estudiantes de la Universidad de Alcalá. Alcalá de Henares: Servicio de Publicaciones. Universidad de Alcalá.

\footnotetext{
${ }^{26}$ IRACHETA FERNÁNDEZ, F. (2012), "Sobre dignidad y eutanasia voluntaria: tres aproximaciones morales (II parte)", Límite. Revista de Filosofía y Psicología, 25, pp. 29, 30, [en línea] https://www.redalyc.org/pdf/836/83624079003.pdf.

${ }^{27}$ IRACHETA FERNÁNDEZ, F. (2012), pp. 31, 32.

${ }^{28}$ SANDEL, M. J. (2013), Justicia ¿Hacemos lo que debemos? Barcelona: Debolsillo, pp. 154- 158.

${ }^{29}$ SANDEL, M. J. (2013), pp. 154-158.

${ }^{30}$ SANDEL, M. J. (2013), p. 157.
}

70 REJIE Nueva época. Núm. 26-Enero 2022 - In memoriam José Calvo González 
- FARIÑAS, Ma . J. (2000), "El conocimiento científico del Derecho", en Peces-Barba, G., Fernández, E., de Asís, R.: Curso de Teoría del Derecho. Madrid: Marcial Pons, segunda edición.

- GARCÍA SAN MIGUEL, L. (2003), Los fundamentos del Derecho (penúltimos apuntes). Madrid: Dykinson.

- GARRIDO GÓMEZ, M. ${ }^{\mathrm{a}}$ I. (2013), "La innovación en la docencia y el aprendizaje de la Filosofía del Derecho por medio del método de casos", REJIE: Revista Jurídica de Investigación e Innovación Educativa, 8, pp. 25-42, [en línea] https://revistas.uma.es/index.php/rejienuevaepoca/article/view/7749/7263.

- IRACHETA FERNÁNDEZ, F. (2012), "Sobre dignidad y eutanasia voluntaria: tres aproximaciones morales (II parte)", Límite. Revista de Filosofía y Psicología, 25, pp. 25-39, [en línea] https://www.redalyc.org/pdf/836/83624079003.pdf.

- JONES, D. (dir.) (2011), Código fuente. Vendôme International.

- MARGALEF GARCÍA, L. ([s. d.]), Estrategias metodológicas. [Alcalá de Henares]: Vicerrectorado de Planificación Académica y Profesorado. Dirección de Formación del Profesorado Universitario. Universidad de Alcalá. [Dosier].

- NOZICK, R. (1974), Anarquía, Estado y utopía. México: Fondo de Cultura Económica.

- RUIZ RESA, J. D. (2017), Innovación docente y nuevas tecnologías. El derecho en la economía del aprendizaje. Valencia: Tirant lo Blanch.

- SÁNCHEZ MUÑOZ, J. M. (2018), "Informe sobre la película «Descifrando Enigma»", Revista "Pensamiento Matemático", VIII / 2, pp. 191-199, [en línea] https://dialnet.unirioja.es/servlet/articulo?codigo $=6636704$.

- SANDEL, M. J. (2013), Justicia ¿Hacemos lo que debemos? Barcelona: Debolsillo.

- TYLDUM, M. (dir.) (2015), The Imitation Game (Descifrando Enigma). Black Bear Pictures.

- UNIVERSIDAD DE ALCALÁ (s. d.), Medidas de prevención COVID-19 Coronavirus. Protégete y protege a los demás. Universidad de Alcalá, [en línea] https:/derecho.uah.es/facultad/documentos/medidas-prevencion-COVID.pdf.

- UNIVERSIDAD DE ALCALÁ (s. d.), Plan de actuación de la Universidad de Alcalá para la adecuación de la actividad universitaria en el curso 2020-2021, [en línea] https://www.uah.es/export/sites/uah/.galleries/documentos/Plan-deActuacion_Curso-2020-2021.pdf.

- WACHOWSKI, J. S., WACHOWSKI, L. (dir.) (1999), Matrix. Warner Bros.

- WACHOWSKI, J. S., WACHOWSKI, L. (dir.) (2004), The Roots of the Matrix. Warner Bros.

- ZAPATERO, V., GARRIDO GÓMEZ, M. ${ }^{a}$ I., ARCOS RAMÍREZ, F. (2010), El Derecho como proceso normativo. Lecciones de Teoría del Derecho. Alcalá de Henares: Servicio de Publicaciones. Universidad de Alcalá, $2^{a}$ edición. 